\title{
L'influence de la «théorie du sujet pluriel » de Margaret Gilbert dans les sciences sociales
}

\section{Sébastien Urbanski}

\section{(2) OpenEdition}

\section{Journals}

Édition électronique

URL : http://journals.openedition.org/ress/2725

DOI : $10.4000 /$ ress. 2725

ISSN : 1663-4446

Éditeur

Librairie Droz

Édition imprimée

Date de publication : 6 mai 2014

Pagination : 225-252

ISBN : 978-2-600-01829-6

ISSN : 0048-8046

Référence électronique

Sébastien Urbanski, «L'influence de la « théorie du sujet pluriel » de Margaret Gilbert dans les sciences sociales », Revue européenne des sciences sociales [En ligne], 52-1 | 2014, mis en ligne le 12 mai 2014, consulté le 19 avril 2019. URL : http://journals.openedition.org/ress/2725; DOI : 10.4000/ress.2725 


\title{
L'INFLUENCE DE LA «THÉORIE DU SUJET PLURIEL » DE MARGARET GILBERT DANS LES SCIENCES SOCIALES
}

SÉBASTIEN URBANSKI

Université Lumière Lyon-2 sebastien.urbanski@gmail.com

Résumé. Si la pensée de Margaret Gilbert est fondamentale en philosophie sociale, elle reste encore assez peu connue en sociologie, en économie, ou en anthropologie. Mais cette situation semble changer, car des sociologues, économistes et anthropologues de premier plan se réfèrent aujourd'hui à l'œuvre de Gilbert et à sa théorie du «sujet pluriel». L'article fait le point sur l'influence interdisciplinaire de cette théorie, puis traite des principales critiques qui lui sont adressées. Au terme du parcours, il apparaît que l'œuvre de Gilbert a un authentique écho dans les sciences sociales, même si elle gagnerait encore à être discutée et complexifiée.

Mots-clés: action conjointe, croyance, groupe, holisme, Margaret Gilbert, socialité.

\begin{abstract}
Whereas Margaret Gilbert's thought is of primary importance in social philosophy, it remains relatively unknown in the fields of sociology, economics, or anthropology. However, this situation seems to change, because leading sociologists, economists and anthropologists refer to Gilbert's work and her "plural subject theory". The article considers the interdisciplinary influence of this theory, and deals with the main critical arguments addressed to it. Finally, I argue that Gilbert's achievement has a genuine influence in social sciences, although it should still be challenged and complexified.
\end{abstract}

Keywords: belief, group, holism, joint action, Margaret Gilbert, sociality. 
Bien que la «théorie du sujet pluriel» de Margaret Gilbert relève d'abord de la philosophie, elle a été remarquée par des chercheurs appartenant à d’autres disciplines, notamment la sociologie, l'économie, et la psychologie du développement (qui elle-même peut être considérée comme une branche de l'anthropologie). Le but de cet article est d'abord de faire le point sur la réception du travail de Gilbert dans ces trois disciplines. Ensuite, il s'agira de repérer les critiques qui lui sont faites sur les terrains philosophique et sociologique. Le parcours sera ainsi balisé par les réflexions que des sociologues (Bernard Conein, Laurence Kaufmann, Dany Trom), des économistes (Philippe Batifoulier, André Orléan), des philosophes (Alban Bouvier, Anthonie Meijers, Hans Bernhard Schmid), et un psychologue du développement (Michael Tomasello) ont émises sur la pensée manifestement influente de Gilbert. Ceci dans le but de fournir au lecteur un «plan d'orientation» sur une œuvre parfois difficile à saisir en raison même de son impact interdisciplinaire. Dans un premier temps, on présentera la «théorie du sujet pluriel» de la façon la plus concise possible. C'est ensuite, lors de l'examen de sa réception dans les sciences sociales, que ses diverses déclinaisons et ramifications seront étudiées.

Comme le suggèrent les philosophes Vincent Descombes et Michael Bratman, l'exemple de la «promenade ensemble» constitue une bonne introduction à la pensée de Gilbert. Descombes note qu'une question centrale est de savoir «ce qui distingue [...] deux personnes [faisant une promenade ensemble] de deux promeneurs qui se trouvent sur le même chemin, mais qui y font l'un et l'autre (au même endroit et au même moment) une promenade personnelle $\gg^{1}$ (Descombes, 2000, p. 43). Ou bien, dans une autre formulation, celle de Bratman: «supposez que vous et moi marchons ensemble sur la $5^{\mathrm{e}}$ avenue, mais qu'il y a aussi une autre personne ["l'étranger"] marchant à côté de nous», sachant que «nous avançons tous les trois sans nous heurter mutuellement ». L'énigme est alors la suivante : «Qu’est-ce qui distingue votre relation à 
moi (et ma relation à vous), dans le cadre de notre activité partagée, de la relation qu’a chacun d'entre nous à l'égard de l'étranger? »(Bratman, 2009, p. 50)².

On pourrait penser, en suivant une conception individualiste, que deux personnes dont on peut dire qu'elles «marchent ensemble» ont un objectif personnel partagé et le savent mutuellement. Dans le cas d'une promenade de vous avec moi, les états mentaux impliqués seraient les suivants: I) je sais que vous avez pour objectif personnel de marcher avec moi ; 2) vous savez que j’ai pour objectif personnel de marcher avec vous; 3) vous savez et je sais que les propositions (I) et (2) sont vraies. Cependant, Gilbert conteste cette interprétation. «Imaginons que Sue Jones est sortie pour se promener seule le long d'Horsebarn Road. Elle s'aperçoit tout à coup que quelqu'un d'autre - un homme vêtu d'un manteau noir - a commencé à marcher à ses côtés, à un pas environ » (Gilbert, 2003a [1990], p. 48). Sue est contente que cet homme soit là, car «elle l'a reconnu ; il s'agit de Jack Smith et il lui plairait de faire sa connaissance » (ibid.). Jack Smith est dans les mêmes dispositions. Ainsi, l'objectif personnel de chacun est de marcher en compagnie de l'autre. Cependant, comment cet objectif personnel partagé pourrait-il être en soi porté au rang de savoir commun?

Il faudrait peut-être supposer que Jack dise, de façon un peu bizarre : «Mon objectif en ce moment précis est de continuer à marcher en votre compagnie», et que Sue réponde «Et mon objectif est de continuer à marcher en votre compagnie ». Mais ici, bien qu'il soit de savoir commun que l'objectif personnel de chacun est partagé, «l'élément crucial requis pour établir que les parties en présence se promènent ensemble semble manquer». Certes «chacun est conforté dans sa présomption que l'autre [...] fera ce qu'il ou elle peut pour s'assurer que l'objectif personnel partagé est atteint» (ibid., p. 56). Mais aucun ne semble «fondé à formuler des reproches à l'encontre de l'autre pour n’avoir

2 Ces exemples des «marcheurs» sont similaires à l'exemple wébérien des deux cyclistes qui s'évitent mutuellement (Weber, 1995 [1921], p. 53). Mais tandis que Weber qualifie «la tentative d'éviter l'autre» (ibid.) d'action sociale, Gilbert insiste sur la différence entre le fait d'éviter l'autre et le fait de marcher ensemble. Selon elle, les actions sociales à la Weber ne sont pas au principe des groupes sociaux: par exemple, des cueilleurs de champignons faisant attention à ne pas gêner les cueilleurs alentour, et veillant même à autoriser leurs voisins à venir sur «leur» morceau de forêt, ne forment pas pour autant un groupe social (voir Gilbert, 1989, p. 34-44). 
pas fait ce qu'il peut pour atteindre l'objectif» (ibid.). Tout au plus, «Jack sera comme "autorisé à s'attendre" à ce que Sue l'interpelle s'il prend sans savoir de l'avance, et Sue sera "autorisée à s'attendre” à ce qu'il ne s'étonne pas de cette façon de procéder » (ibid.). Or, c'est précisément cette possibilité d'émettre des reproches qui caractérise une situation dans laquelle deux personnes marchent ensemble ${ }^{3}$. Ainsi, le modèle de l'objectif personnel partagé ne convient pas pour deux raisons: d'abord, l'expression de cet objectif partagé risque d'être assez irréaliste (les déclarations de Jack et Sue sont plutôt bizarres) ; ensuite, le savoir commun portant sur cet objectif partagé ne suffit pas à faire naître des obligations, et corrélativement des droits à émettre des reproches.

Marcher ensemble n'est donc pas, selon Gilbert, une activité fondée sur l'objectif personnel partagé des participants, mais c'est «une activité dont l'objectif est l'objectif d'un sujet pluriel [...]. Autrement dit, le fait de se promener implique un objectif "nôtre" par opposition à deux objectifs "miens" ou plus » (ibid., p. 63). Par exemple : Jack Smith demande à Sue si elle est bien Sue Jones et si elle verrait un inconvénient à ce qu’il l’accompagne; Sue répond «Non, cela pourrait être agréable. J’apprécierais d’avoir de la compagnie». Dans ce cas, chacune des parties a exprimé «une volonté de constituer avec l'autre un "sujet pluriel” de l'objectif consistant à se promener en compagnie l'un de l'autre» (ibid., p. 58). Il s'agit là de «l'unification [binding together] d’une série de volontés individuelles susceptible de constituer une "volonté plurielle" unique, consacrée à un objectif particulier» (ibid.). Les parties prenantes sont alors conjointement engagées (ou co-engagées), telles un seul corps (as a body), à réaliser l'objectif de marcher ensemble - ou, dans d'autres contextes, de voyager ensemble, de mener ensemble une enquête sur un meurtre, etc.

L’idée de Gilbert ainsi résumée semble assez banale, mais c'est peut-être son évidence phénoménologique qui lui assure une audience interdisciplinaire. Si nous ressentons une certaine justesse dans ce que dit Gilbert, c'est peut-être parce que :

3 Par exemple, Anna et Joseph marchent ensemble et ce dernier «s'arrête soudain de marcher et s'assoit sur un banc. Anna le lui reproche et Joseph comprend qu'Anna est en position de lui faire des reproches vu leur intention conjointe» (Gilbert, 2003a [1990], p. 34). 
- l'intention conjointe est une capacité psychologique qui nous distingue en tant qu'êtres humains (Tomasello, 2009);

- quand nous faisons «quelque chose ensemble», il y a souvent en jeu «quelque chose de plus» que la rationalité stratégique, même d'un point de vue économique (Orléan, 2004) ;

- quand nous voulons inviter quelqu'un, nous prenons des précautions oratoires importantes pour minimiser notre engagement personnel et s'assurer du caractère conjoint de l'action à accomplir (Conein, 2005) ;

- nous sentons bien que, faisant partie d'un «sujet pluriel», il nous est difficile de soutenir publiquement une croyance contredisant celle sur laquelle il y a eu co-engagement (Bouvier, 2004);

- le contractualisme du modèle de Gilbert paraît réaliste pour le cas de collectifs formés sur la base du consentement de leurs membres; de plus, il est politiquement attractif en posant la question du pouvoir d'action des individus dans la constitution des collectifs (Kaufmann, 2010).

Ces points seront développés dans la suite (section I). Ce qui n'exclut pas, évidemment, que le modèle de Gilbert puisse avoir de sérieuses limites (section 2) :

- il n'est pas sûr que l'engagement conjoint dans un cours d'action soit suffisant, ni nécessaire, pour former un groupe social (Goffman, 1972);

- le paradigme du groupe social n'est pas la dyade mais la triade, car celui-ci émerge quand une paire d'individus (humains ou primates non-humains) trace une frontière en créant de la distance à l'égard d'un acteur tiers (Conein, 2007);

- il n'est pas sûr que le co-engagement soit, ainsi que le prétend Gilbert, au principe de croyances collectives au sens strict (Meijers, 2002);

- les conditions pour que le co-engagement soit assumé par tous les membres d'un groupe ne sont pas toujours présentes (Bouvier, 2010) ${ }^{4}$. 


\section{I.PROLONGEMENTS INTERDISCIPLINAIRES DE LA THÉORIE DU SUJET PLURIEL}

En premier lieu, nous aborderons la question des interactions les plus simples et les plus «épurées»: dispositif expérimental de Tomasello et al. (2009) en psychologie, réflexions de Batifoulier (200I) et Orléan (2004) en économie. Puis, on envisagera des cas sociologiques plus situés et complexes (analyse conversationnelle de Conein, analyse d'écoles scientifiques chez Bouvier). Enfin, nous aborderons la question de savoir dans quelle mesure le modèle de Gilbert est pertinent pour rendre compte de collectifs à grande échelle (Kaufmann). Au terme de ce parcours, le passage à la section 2 - c'est-à-dire aux critiques portées à la théorie du sujet pluriel - devrait se faire assez naturellement : en effet, plus on s'approche du niveau macro, plus les limites de la pensée gilbertienne se font sentir.

\section{I.I.PSYCHOLOGIE DU DÉVELOPPEMENT (ET ANTHROPOLOGIE)}

Comme mentionné au début du présent article, une idée fondamentale de Gilbert est que des personnes co-engagées sont fondées à émettre des reproches à l'encontre de tout membre qui ne ferait pas ce qu'il peut pour atteindre l'objectif commun. Si cela est juste, alors on peut émettre l'hypothèse que des enfants s'étant co-engagés avec un adulte dans un jeu devraient I) réagir au comportement de l’adulte s'il abandonne le jeu sans prévenir ; 2) prévenir l’adulte qu’ils quittent le jeu (si tel est leur désir). C’est ce qu’une étude de psychologie expérimentale conduite par Gräfenhain, Behne, Carpenter et Tomasello cherche à établir, en analysant la «compréhension du co-engagement » (understanding of joint commitment) chez les enfants de 2 à 4 ans (Gräfenhain et al., 2009).

Le dispositif expérimental mis en place est très sophistiqué, mais on peut le résumer comme suit. Dans la condition avec co-engagement, un adulte joue à un jeu pouvant être pratiqué aussi bien seul quà deux. Ensuite, il demande à l'enfant: «Oh, [nom de l'enfant], tu joues avec moi?». Quand ce dernier donne son accord (verbalement ou non), l'adulte dit : «Alors allons jouer à [nom du jeu] ensemble !». Par contraste, dans la condition sans co-engagement, l’adulte, 
après avoir joué seul, s'éloigne et un autre adulte dit alors à l'enfant: «Alors, [nom de l'enfant], maintenant tu peux jouer!». Dès que l'enfant commence à jouer, le premier adulte le rejoint en disant: «Oh, c'est amusant. Je vais jouer à [nom du jeu]!». Enfin, dans les deux conditions expérimentales (avec et sans co-engagement), l’adulte s'arrête de jouer sans prévenir. Dans la condition avec co-engagement, les enfants essaient majoritairement de réengager l'adulte dans le jeu, par exemple en les regardant tout en pointant du doigt en direction du jeu. Ceci suggère qu'ils ont interprété l'action comme étant conjointe, ce qui leur donne le droit de faire une demande à l'adulte. Dans la condition sans co-engagement, les enfants continuent en général à jouer en solitaire. Mais les enfants de deux ans ne font pas de différence significative entre les deux conditions, ce qui suggère que le sens du co-engagement émerge plutôt vers trois ans.

Cette étude permet de faire face à des hypothèses concurrentes: par exemple, peut-être que les enfants essaient de réengager l'adulte tout simplement parce que ce dernier semble plus «sociable» dans la condition avec co-engagement? Cette interprétation est possible mais peu plausible: en effet, durant les phases d'interruption du jeu, les enfants ont parlé à l'adulte dans les deux conditions, et ce dans les mêmes proportions (Gräfenhain et al., 2009, p. I436). Cependant, une limite de l'expérience est qu’on ne voit pas précisément si le comportement des enfants relève d'un objectif personnel partagé (modèle de Bratman) ou de l'objectif d'un sujet pluriel (modèle de Gilbert). En effet, les enfants sont-ils seulement autorisés à s'attendre que l'adulte ne quitte pas le jeu (objectif personnel partagé) ou se sentent-ils «floués » (sujet pluriel)? Même si la seconde hypothèse était vraie, cela n’est pas vérifiable dans le cadre de ce dispositif expérimental, car il y a de toute façon peu de chances que des enfants de 2 à 4 ans émettent des reproches à un adulte qu'ils ne connaissent pas. Cela dit, c'est bien à Gilbert et non à Bratman que les auteurs se réfèrent lorsqu'ils abordent la question des droits afférents à une action conjointe (ibid., p. I430) ${ }^{6}$.

ensemble, pouvant désigner une action conjointe et le sujet pluriel approprié. Le terme avec - utilisé dans l'expression précédente «tu joues avec moi?» - est similaire: faire une promenade avec quelqu'un, pour reprendre l'exemple favori de Gilbert. Voir StavoDebauge (2010) qui mentionne l'apport de Gilbert.

6 L'équipe de Tomasello est amenée à faire une étude complémentaire, dans laquelle c'est l'en- 
Cette étude de psychologie du développement met l'accent sur un élément indispensable à la constitution des sujets pluriels: le savoir commun (common knowledge). En effet, il n'y a action conjointe que si tel individu sait que l'autre est co-engagé et vice-versa. Or, si dans l'étude susmentionnée la situation d’invitation n'est pas interprétée par les enfants de deux ans comme donnant lieu à la constitution d'un sujet pluriel, c'est parce que le savoir commun n'est pas en place : les capacités psychologiques requises semblent émerger vers trois ans ${ }^{7}$. Par ailleurs, la question connexe du degré d'explicitation est posée (Tomasello, 2009, p. 67) : les enfants semblent avoir besoin de signes clairs (utilisation des mots «avec» ou «ensemble», etc.) indiquant la volonté de co-engagement de l'adulte. On verra plus loin que la condition de savoir commun est parfois difficile à réaliser dans des situations moins expérimentales, justement en raison du caractère peu explicite de certains co-engagements supposés, ce qui requiert peut-être d'amender le modèle du sujet pluriel (infra, section 2.3) .

\section{I.2. ÉCONOMIE}

En économie, la notion de savoir commun sert à comprendre l'émergence et le maintien d'un phénomène appelé convention, c'est-à-dire :

une régularité de comportement $\mathrm{R}$ au sein d'une population $\mathrm{P}$ telle que : I) tous les membres de la population se conforment à $\mathrm{R}$; 2) chacun croit que tous les autres membres de $\mathrm{P}$ se conforment à $\mathrm{R}$ [condition de savoir commun] et 3) trouve dans cette croyance une bonne et décisive raison pour se conformer à $R$; 4) par ailleurs, au moins une autre régularité R' vérifiant les conditions précédentes aurait pu prévaloir (Orléan, 2004, p. 13).

fant (et non plus l'adulte) qui quitte le jeu. On ne la présentera pas ici par manque de place.

7 Sur les capacités psychologiques supposées par modèles de l'intentionnalité collective, notamment le raisonnement récursif, voir le point de vue critique de Pacherie (20ll).

8 Cela dit, Gilbert reconnaît que la condition de savoir commun n'est pas toujours réalisée, notamment quand la référence subjective à un co-engagement est tendancieuse: "Jill pourrait par exemple s'offenser de la question de Jack "si nous allions nous promener?", bien qu'elle n'accorderait pas d'importance à [... "ça vous dirait de faire un tour?", et des formules semblables. L'une des explications à cette sensibilité réside dans le fait que "nous" a spontanément été interprété comme sous-entendant l'existence d'un sujet pluriel [...] alors que, d'après l'interlocutrice, il n'existe nul sujet pluriel» (Gilbert, 2003b [1999], p. 166). Cette attention au point de vue subjectif est explicitement inspirée de Max Weber (ibid., p. 29-43). 
Cette définition trouve son origine dans les travaux de David Lewis (2002), un logicien dont s'inspira également Gilbert. Dans ce cadre, une convention émerge suite à des décisions rationnelles dans un jeu, au sens de la théorie des jeux. Par exemple: deux individus perdus dans New York cherchent à se retrouver. Chacun doit donc imaginer l'endroit que l'autre choisira pour se retrouver, et cet endroit ne sera approprié que si l'autre le choisit aussi. Il est possible que les individus décideront d'aller à la gare centrale, parmi une multitude d'autres endroits possibles. Cette solution, si elle survient, est une convention à la fois arbitraire et rationnelle. Arbitraire car la gare centrale n'est pas en soi un lieu plus approprié qu'un autre; rationnelle car aller à la gare centrale a un sens si l'on pense que l'autre aussi s'y rendra: je vais «à la gare centrale $[. .$.$] uniquement si l'autre pense que j'y vais et si je pense qu'il pense$ cela » (Urrutiaguer, Batifoulier, Merchiers, 200I, p. 74) .

Cependant, selon Gilbert, cette conception ne rend pas compte de ce que nous entendons, dans la vie de tous les jours, par convention - alors même que Lewis entendait explorer «notre concept ordinaire et établi» de ce phénomène (Lewis, cité par Gilbert, 2008a, p. 6). Imaginons des individus parlant de la façon suivante: «La convention dans notre département est de nous habiller formellement pour les réunions du département». Un jour, Joe vient à une réunion en tee-shirt et en jeans. Molly pourrait bien le lui reprocher. En tout cas, « elle est en position de le faire [...]. Elle pourrait [...] justifier son reproche en remarquant que la convention en question est en vigueur au sein du département» (Gilbert, ibid., p. 7). Or, dans la conception lewisienne selon laquelle les membres d'une population $\mathrm{P}$ se conforment à une convention $\mathrm{R}$ en raison de la croyance selon laquelle tous les autres membres de $\mathrm{P}$ se conforment à $\mathrm{R}$ (voir supra), aucun reproche de ce genre n'est possible. Certes, si June venait en tenue décontractée, «Bob pourrait être surpris, même déçu [...], [ou encore] juger qu'elle agit mal. Après tout, elle agit en contradiction avec les préférences de la plupart des membres. Néanmoins, elle ne l’a pas offensé de telle sorte qu'il serait maintenant en position de la réprimander» (ibid., p. ıo). 
En somme, notre conception ordinaire de ce qu'est une convention inclut l'idée d'une offense possible. Le modèle du sujet pluriel s'accorde avec cette conception, et permet de formuler une nouvelle définition. Soit A une action conforme à une convention donnée. On peut dire que $\mathrm{X}$ offense $\mathrm{Y}$ en manquant de faire A en une certaine occasion «seulement si X devait [owed] A à Y en cette occasion, sachant que celui à qui on doit une action [one who is owed an action] est en position de la demander comme si elle était en quelque sorte la sienne» (ibid.). Or, soutient Gilbert, pour que l'action A de X soit due à Y comme si elle était la sienne, il faut au préalable que $\mathrm{X}$ et $\mathrm{Y}$ aient formé le sujet pluriel de la convention prescrivant de faire A. L'action A est alors autant l'action de X (en tant qu'il en sera peut-être l'auteur, vu qu'il la doit à Y) que l'action de Y (en tant qu'elle lui est due). D’où la définition gilbertienne de la convention: «Une population $\mathrm{P}$ a une convention de conformité à une régularité de comportement $\mathrm{R}$ dans les situations de type $\mathrm{S}$ si et seulement si les membres de $\mathrm{P}$ acceptent conjointement, en ce qui les concerne, le principe : il faut se conformer à R» (ibid., p. II-I2). Les actions conformes à $\mathrm{R}$ sont alors, avant qu'elles ne se produisent, dues aux autres parties. Si une partie menaçait de ne pas se conformer à $\mathrm{R}$, alors n'importe qui d'autre au sein de P pourrait solliciter la conformité en disant «Je demande que vous me donniez ce qui est mien», sachant que «ce qui est mien» fait ici partie de ce qui est «nôtre».

Comme le montre Orléan - qui se base toutefois sur un autre exemple ${ }^{10}-$ le modèle de Gilbert permet d'expliciter la notion de convention légitimée (par opposition aux conventions émergeant uniquement de décisions rationnelles dans un jeu), en rejoignant ainsi Max Weber. Selon Weber, la convention est un «ordre légitime» dont la stabilité est «garantie par la réprobation de tout écart» (Weber, cité par Orléan, 2004, p. I4). Or, c’est bien ce phénomène de réprobation que Gilbert cherche à saisir. En conséquence, la théorie du sujet pluriel constitue un apport potentiel à toute analyse économique voulant aller au-delà de «la conception traditionnelle réduisant les interactions humaines au seul principe de la rationalité instrumentale» (Orléan, ibid., p. 20).

I0 Celui du «dilemme de l'hôte» (Gilbert, 2003a [1990], p. II3-II5), qui constitue également une critique à l'égard de Lewis. 
L'apport de Gilbert est d'autant plus important, du point de vue de l'économie des conventions, que Lewis est un précurseur de la théorie des conventions: sans le «petit livre [...] du logicien américain David Lewis (Convention: a philosophical study, 1969) [...] la théorie des conventions n'aurait pas vu le jour» (Favereau, 200I, p. 5). Cependant, certains économistes ne manquent pas de relever les failles du raisonnement de Gilbert. En particulier, la solution en termes de sujet pluriel tend à suggérer que seuls les collectifs (les sujets pluriels) peuvent avoir des conventions. Or, «suivre une convention n'implique pas forcément l'existence d'un collectif. Il existe une convention dans une campagne anglaise où la priorité de passage sur un petit pont est donnée à ceux qui viennent du Nord. Si des touristes respectent cette convention, ils ne deviennent pas pour autant un collectif» (Urrutiaguer, Batifoulier, Merchiers, 200I, p. 90). On verra plus loin que l'affirmation selon laquelle tous les sujets pluriels sont des groupes sociaux constitue en effet un point faible de la théorie de Gilbert (infra, section 2.I).

\section{I.3. SOCIOLOGIE}

\section{I.3.I. Niveau des interactions: analyse de conversations}

Contrairement à de nombreuses interprétations qui en sont faites, le modèle de Gilbert vise à saisir un phénomène qui n'est pas de l'ordre de la promesse. Dans une promesse, chacun se lie unilatéralement à l'objectif en question; par ailleurs, celui qui promet ne peut prétendre à aucune revendication à l'égard du destinataire de la promesse (Gilbert, 2003a [1990], p. 60). Par contraste, un sujet pluriel suppose un engagement conditionnel tel que «n'importe qui est engagé $[\ldots]$ lorsque chacun est engagé. Toutes les volontés sont ainsi liés simultanément et de façon interdépendante» (ibid.). Ceci pourrait sembler à première vue assez mystérieux, mais une analyse de conversation réalisée par Bernard Conein permet d'éclairer concrètement ce point.

Conein conteste la pertinence de la théorie austinienne des actes de langage selon laquelle une invitation se produit au moyen d'un énoncé performatif dont le modèle est «celui d'un acte individuel et d'un engagement personnel du locuteur, qui, dans le cas de la promesse et de l'invitation, engage uniquement le destinateur » (Conein, 2005, p. I29). Il semble en effet que lors d'une invitation, 
le destinataire est engagé avant même d'avoir notifié verbalement son accord ou son refus. Un indice allant dans ce sens est que l'alternative qui s'offre au destinataire n’est jamais symétrique: comme l’a remarqué Harvey Sacks, l’acceptation a généralement un caractère «direct et ouvert», tandis que les refus «sont plus souvent indirects et mitigés» (ibid., p. I20). Précisément, la théorie du sujet pluriel vise à rendre compte de ce genre « d'engagement sans accord » : un co-engagement au sens de Gilbert ne requiert pas forcément d'accord. Par exemple, Joe fait signe à Lisa depuis la piste de danse de s'approcher et Lisa s'avance pour l'y rejoindre. Chacun est alors «le sujet pluriel du fait de danser ensemble», alors qu'il n'y a pas eu d'accord, même tacite: «ce cas de figure ne semble pas $[\ldots]$ faire appel à un accord tacite $[\ldots]$. Il ne serait pas exact de la part de l'une ou l'autre des parties de dire à la seconde : vous avez donné votre accord» (Gilbert, 2003b [1999], p. 154)". C'est ici que Gilbert rejoint Goffman, pour qui «un engagement conjoint précède les acceptations verbalisées d'une action commune» (Conein, 2005, p. I22).

Il est alors possible d’expliquer la fréquence des énoncés indirects consistant par exemple à interroger l'autre sur son désir («tu veux faire un truc?») au lieu d'utiliser la forme directe («je t’invite»). Selon la théorie des actes de langage, les énoncés indirects sont utilisés pour des raisons de politesse: «la motivation principale - sinon la seule - qui conduit à employer ces formes indirectes est la politesse » (Searle, 1982 [1979], p. 90). Or, cette explication néglige les contraintes sociales, notamment les modalités de co-orientation entre les personnes, qui caractérisent les situations d'invitation. Selon Conein, une invitation requiert surtout que le destinateur s'assure du caractère authentiquement conjoint de l'engagement à venir. C'est ce qui explique les formules d'ouverture du type «qu'est-ce que tu fais, là, en ce moment? », qui atténuent, dès le début de l'échange, la part d'engagement individuel du locuteur. C'est ce qui explique également la réitération des interrogations sur le désir du destinataire, du type «AI Si tu veux je t'invite; B2 ouais ouais; A3 tu voudrais?; B4 ouais». À terme, ces procédures permettent de faire émerger une action 
véritablement conjointe, c'est-à-dire qui ne soit pas «une simple agrégation de deux engagements personnels» (Conein, 2005, p. I29). Ainsi, Gilbert semble viser juste quand elle dit qu'une «liaison simultanée et interdépendante des volontés » (supra) suppose un engagement conditionnel: prendre un café ensemble ou dîner ensemble requiert de manifester à l'autre qu'on est engagé à la condition que chacun le soit. Une action véritablement conjointe ne peut même pas se décrire comme impliquant «une personne qui dirait en fait: “Vous pouvez me considérer comme m’étant engagé dès lors que vous vous êtes engagé”, en laissant à l'autre personne l'initiative d'un engagement unilatéral» (Gilbert, 2003a [1990], p. 60). En effet, comme le montre Conein, «l'interrogation sur le désir de l'autre concerne autant A [le destinateur] que B [le destinataire], puisqu'il arrive que ce soit B qui tient à s'assurer de l'engagement de A avant d'accepter l'invitation»(Conein, 2005, p. I24).

Les linguistes A. Carassa et $\mathrm{M}$. Colombetti proposent des réflexions similaires à celles de Conein, en recourant également à la thèse gilbertienne d'une «liaison simultanée et interdépendante des volontés ». Dans le cas d’une requête entre deux personnes qui se connaissent, le processus est selon eux le suivant: A rend disponible une affordance ${ }^{12}$ («j’aurais besoin de quelques livres») qui laisse à B la possibilité d'interpréter cela comme une requête («ok, donne-moi la liste») ou une demande moins forte («essaye Amazon, les frais de port sont gratuits»). Selon la réponse de $\mathrm{B}$, les parties seront co-engagées sur le sens des énoncés exprimés au tour de parole précédent. Pour les auteurs, un avantage de ce modèle est de ne pas supposer que A connaît clairement son intention communicative dès le départ : en effet, c'est seulement après les réponses de $B$ que le sens de ce que dit A est établi. Plus précisément, les parties construisent ensemble un sens conjoint ou joint meaning, qui peut éventuellement être différent du sens du locuteur ou speaker's meaning. Par exemple,

Albert dit à Barbara : «Je crois que je vais aller marcher», sans aucune intention de l'inviter [...]. Barbara répond: «Désolée Albert, je suis trop occupée ». Barbara a considéré l'énoncé d'Albert comme une invitation. Albert pourrait prendre en charge [take up] l'énoncé de Barbara, en sous-entendant par consé- 
quent que son énoncé initial était une invitation; il pourrait dire par exemple : «Dommage. Bon, je reviens bientôt». À ce point de la conversation, le sens conjoint [joint meaning] de l'énoncé initial d'Albert est que c'était une invitation (Carassa, Colombetti, 2009, p. 1851).

Le point crucial est qu'Albert, malgré sa croyance personnelle sur le sens de son énoncé initial, est co-engagé à croire que ce dernier était une invitation. En effet, supposons que Barbara change d'avis et dise : «Oh, en fait, je crois que mon travail peut attendre. Mais seulement une courte marche, ok?». Albert ne peut pas répondre que son intention était de marcher seul sans violer un co-engagement. Ceci rejoint l'intuition durkheimienne de Gilbert selon laquelle «une croyance collective que $p[. .$.$] ne doit pas être confondue avec ce qu'on appelle$ la croyance commune ou mutuelle [...]. Les croyances collectives $[\ldots]$ sont logiquement indépendantes de ce que les agents en question croient personnellement » (ibid., 20I I, p. I057). C’est ce point que nous voudrions maintenant développer.

\section{I.3.2. Niveau des groupes: analyse de croyances collectives}

Jusqu'ici, nous avons suggéré que le modèle de Gilbert est pertinent pour analyser des interactions dans des contextes d’action bien définis : enfants qui jouent, individus suivant une convention légitimée dans un contexte économique, individus proposant une invitation. Mais Gilbert tente de dépasser ce niveau micro (ou «méso») pour rendre compte de «collectifs à grande échelle» (Kaufmann, 2oı, p. 343). Pour cela, elle se réfère à Durkheim et à son idée selon laquelle le fait social I) est inhérent au groupe social; et 2) exerce une contrainte sur les individus. La notion de croyance est privilégiée pour clarifier cette problématique complexe, et permet de formuler deux thèses que Durkheim ne renierait probablement pas : I) «Le caractère général d'une croyance au sein d'un groupe n'est pas une condition suffisante pour que celle-ci puisse être tenue pour une croyance de groupe »; et 2) «lorsqu'un groupe soutient une certaine croyance, il s'ensuite que certaines obligations s'imposent à ses membres» (Gilbert, 2003a [1990], p. 85, p. 87) ${ }^{13}$.

13 Rappelons que pour Durkheim, les faits sociaux sont «constitués» par «les croyances, les tendances, les pratiques du groupe pris collectivement» (Durkheim, 2005 [1894], p. 8, nous soulignons). 
Il y a bien des cas où ces deux conditions (inhérence au groupe et contrainte) sont remplies. Parfois même, une croyance de groupe peut n'être partagée par aucun membre du groupe. Pour le montrer, on peut comparer les croyances du «Cercle de Vienne» (incluant par exemple Carnap ou Gödel) avec celles de «l'école de Copenhague» (incluant par exemple Bohr ou Heisenberg). Les membres du Cercle de Vienne ont produit un manifeste destiné à exprimer les croyances collectives du groupe. Ces croyances étaient assez générales et vagues pour que chaque membre puisse y adhérer personnellement: «chacun semble tout à fait d’accord avec les idées communes exprimées par seulement trois des membres (Hahn, Neurath et Carnap) [du Cercle de Vienne] » (Bouvier, 2004, p. 39I). Dans ce cas, le groupe exerce une certaine contrainte car chacun des membres ne peut abandonner les idées exprimées dans le manifeste sans fournir une justification aux autres membres. Mais il n'est pas sûr que la croyance soit ici inhérente au groupe : elle semble plutôt avoir les individus pour substrat, puisque chacun adhère personnellement à la croyance du groupe. Un cas plus typiquement durkheimien est celui de l'«école de Copenhague», un groupe dont aucun membre ne partage la croyance collective - du moins si l'on suit l'interprétation de l'historienne des sciences Mara Beller.

En 1927, le physicien Niels Bohr propose une théorie en mécanique quantique censée exprimer le point de vue des membres de l'«école de Copenhague ». Il fallait en effet «une théorie unifiée, capable de contrer l'opposition» (Beller, 1999, p. I43), notamment celle de Schrödinger et Einstein. Dans le but de former un front public uni, les principaux membres de l'école de Copenhague (Bohr, Heisenberg, Pauli et Born) «serrèrent les rangs au Congrès de Côme et au colloque Solvay à Bruxelles en 1927 » (ibid., p. 216). Bohr entreprit de faire une synthèse des points de vue des divers physiciens, mais sa conférence à Côme - publiée par la suite dans Nature - est confuse et difficile à comprendre. C'est que «l'interprétation rendue publique et sur laquelle tous les membres de l'école de Copenhague prétendaient publiquement s'entendre continuait à être, en réalité, l'objet de discussions à l'intérieur 
même du groupe ». (Bouvier, 2002, p. 46) ${ }^{14}$. Il y a donc une différence entre les croyances personnelles (en matière de mécanique quantique) des chercheurs et les croyances collectives, affichées publiquement, de l'école de Copenhague, qui est alors un sujet pluriel: ce n'est pas une simple collection d'individus, pas un simple agrégat, et on peut bien parler de «communauté» scientifique. De plus, aucun individu ne partage personnellement cette croyance collective : en effet, à Côme, Bohr n’exprima même pas sa pensée propre, afin de pouvoir inclure celle de Heisenberg. Par conséquent, la croyance collective ne fut même pas partagée par celui qui pourtant conçut et exprima cette croyance ${ }^{15}$. Dans le cas présent, la conception «durkheimo-gilbertienne» selon laquelle les croyances collectives sont inhérentes au groupe social est pertinente: si aucun membre du groupe n'adhère personnellement à la croyance du groupe, alors cette dernière doit bien, en un certain sens, être inhérente au groupe. Ceci constitue une alternative sérieuse à un strict individualisme méthodologique (Boudon, 1995) en matière de croyances collectives.

\section{I.3.3. La dimension politique des collectifs}

Selon Gilbert, les faits sociaux n'émergent pas forcément d'un contrat, ni d'un accord, ni d'une promesse (Conein, 2005, p. 99, 122). Cependant, les théories contractualistes - celles de Rousseau ou de Hobbes par exemple - forment une sorte d'arrière-plan à la théorie du sujet pluriel, même en ce qui concerne les co-engagements formés sous la contrainte. En effet, pour constituer un sujet pluriel, il suffit que chacune des parties «exprime sa disponibilité » à être co-engagée :

considérons par exemple une famille [...]. Si, alors qu'elle commence à comprendre ce qui se passe autour d'elle, la jeune Betty prend part aux différents co-engagements établis par sa famille, elle n’a probablement guère eu le choix de faire autrement - ses parents ont menacé de la priver de nourriture ou d'un toit si elle leur refusait sa participation. Nonobstant cette absence de libre-arbitre, il

14 II y a cependant d'autres explications possibles (mais pas forcément incompatibles). Selon Chevalley (1985, p. 28I), l'obscurité de la conférence de Côme est due principalement au fait qu'il fallait se distancier d'un langage imprégné d'une ontologique valable uniquement pour les phénomènes macroscopiques: l'abandon nécessaire de l'ontologie physique ordinaire se paie d'une «crise de mots», d'un «vague», d'un «flottement» du langage.

I5 Selon Beller (1999, p. 122), les théories de Bohr et Heisenberg sont incompatibles. 
suffit qu'elle ait manifesté aux siens d'une manière ou d'une autre sa disponibilité à obéir pour se trouver partie prenante [...]. [L]a pression extérieure n'invalide pas l'expression de la disponibilité (Gilbert, 2008b (2006), p. 910).

Ainsi, même le lieu par excellence de la «socialisation primaire» est appréhendé dans une perspective contractualiste. Ceci constitue un point faible de la pensée gilbertienne. En effet, de nombreux collectifs «ne peuvent être le produit d’activités associatives et contractuelles; non seulement ils précèdent ces dernières mais c'est eux qui les rendent possibles en leur assurant d'emblée une "communalité” de principe» (Kaufmann, 20ı, p. 344). Toutefois, le modèle du sujet pluriel reste heuristique pour l'analyse de la constitution des collectifs, quels qu'ils soient. En effet, tout collectif «"en train de se faire" [...] passe par un moment politique $[. .$.$] potentiellement réflexif qui vise à assembler les volontés et à fédé-$ rer les différentes opinions quant à la raison d'être, aux orientations pratiques et aux critères d’appartenance qui sont les siennes» (ibid., p. 353). Ainsi, le modèle du sujet pluriel rend compte de la «collectivisation des collectifs» : les individus co-engagés unifient leurs volontés dans une volonté plurielle unique, qui émerge «par le bas». Mais ce Nous une fois constitué s'appuie sur des médiations tierces, par exemple des représentants ayant explicitement pour tâche de parler et d'agir au nom du groupe, ou des objets (au sens de Latour) fournissant des appuis normatifs aux membres du collectif. Or, ces médiations tierces sont ambivalentes: d'un côté, elles permettent aux individus de «prolonger leur capacité d’agir dans des activités collectives qui leur offrent de nouvelles possibilités d’action (voter, enseigner, etc.)» (ibid., p. 351), mais de l'autre, le tiers «est toujours susceptible de s'émanciper de l'activité conjointe» (ibid. ${ }^{16}$. Enfin, l'existence du collectif dépend de sa reconnaissance ontologique par des instances extérieures ; autrement dit, de sa reconnaissance comme un Ils ou un Eux, par exemple une nation dans l'espace géopolitique des relations internationales. C'est là que le collectif apparait, à rebours de toute description «par le bas», comme une tota-

16 Gilbert (1996, p. 209) occulte quelque peu la réalité des médiations tierces, par exemple quand elle affirme que la croyance d'un gouvernement peut généralement être attribuée à la nation dans son ensemble. Une critique de ce point est formulée par Tuomela (1992), plus attentif aux positions respectives des membres d'un groupe. 
lité concrète court-circuitant «la multiplicité des individus qui le composent» (ibid., p. 355). En somme, le modèle de Gilbert est plausible si on le restitue dans son cadre propre: celui d'une description «par le bas» des collectifs.

Mais, si la théorie du sujet pluriel «se heurte aux limites classiques de tous les modèles contractualistes » (Bouvier, 2004, p. 403), elle permet de poser la question politique de la primauté des volontés individuelles sur celles du collectif, le modèle gilbertien étant une contrepartie analytique de l'idéal démocratique moderne (Kaufmann, 20I0). Du coup, les idées de Gilbert se révèlent pertinentes pour l'étude des collectifs qui se veulent politiques, c'est-à-dire qui posent euxmêmes la question de la transformation des volontés individuelles en une unité d'ordre supérieur. Certes, dès lors que l'on a affaire à des collectifs à grande échelle, l'approche de Gilbert «bute inévitablement sur l'indétermination de l'extension des intentions ou des vouloirs [individuels]» (Trom, 20ı, p. 380). Mais la théorie du sujet pluriel redevient pertinente lorsque «cette indétermination, pourtant inéliminable, est problématisée de l'intérieur du collectif lui-même» (ibid.). En effet, une fois que la question politique de la «transformation du multiple en un» est posée au sein du collectif, «c'est aussi le plan des vouloirs individuels, celui des particuliers dénombrables et des dispositifs de coordination, qui, simultanément, apparaît comme inéliminable » (ibid.).

\section{CRITIQUES PORTÉES À LA THÉORIE DU SUJET PLURIEL}

La section I a déjà permis d'entrevoir certaines limites de la théorie du sujet pluriel. La section présente propose de les identifier plus précisément. Comme nous le verrons, certaines d'entre elles sont relatives et ne requièrent qu'un aménagement du modèle; d’autres sont plus fondamentales.

Les critiques portées à la théorie du sujet pluriel peuvent être classées en trois catégories. Premièrement, on peut questionner sa prétention à rendre compte des groupes sociaux : est-ce que «ceux qui constituent un sujet pluriel, quel qu'il soit, constituent [...] un groupe» (Gilbert, 2003a [1990], p. 106) ? Inversement, tous les groupes sont-ils des sujets pluriels ? Deuxièmement, on peut questionner la tentative de modéliser des groupes sans faire référence à ce 
qui leur est extérieur: l'identité d'un groupe ne dépend-elle pas constitutivement de ses relations avec d'autres groupes? Enfin, on peut questionner la façon un peu abrupte de doter les groupes de croyances : les états mentaux des groupes ne sont-ils pas fondamentalement différents des états mentaux individuels?

\section{I. SUJETS PLURIELS ET GROUPES SOCIAUX}

Bien qu’Erving Goffman soit décédé avant les divers développements sur la théorie du sujet pluriel, on peut trouver dans ses écrits une critique du lien entre action conjointe et groupes sociaux. Or, Gilbert affirme qu'il y a un rapport étroit entre les groupes sociaux et les sujets pluriels, analysables en termes d’action conjointe (ou de croyance collective). Voici une formulation de cette thèse :

tout sujet pluriel est un groupe social [...]. La clé menant aux groupes sociaux est le concept de sujet pluriel [...]. J’ai affirmé que des personnes se promenant ensemble forment un sujet pluriel et qu'il y a quelque raison de supposer que notre concept de groupe social [...] réside dans le concept de sujet pluriel (Gilbert, 2003a [1990], p. 66, p. 7I).

Pour affirmer que même deux personnes se promenant ensemble forment un groupe social, Gilbert fait appel à l'autorité de Simmel. Ce dernier écrit :

[La] socialisation (grossièrement, le processus de formation d'un groupe social) peut avoir des degrés très divers [...] allant de la réunion éphémère en vue d'une promenade jusqu'à la famille [...] de la communauté passagère des clients d'un hôtel jusqu'à la profonde solidarité d'une guilde médiévale. [...] la formation sociologique la plus simple du point de vue méthodologique est la relation entre deux éléments. [...] il s'agit déjà d'une socialisation (Simmel, cité par Gilbert, 2003a [1990], p. 46, p. 69).

Or, si Simmel est bien «un précurseur de l'idée selon laquelle l'action conjointe de deux personnes peut être considérée comme un groupe social» (Conein, 2007, p. 209), cette idée s'inscrit peut-être dans une «tendance à identifier trop facilement les rassemblements orientés (focused gatherings) avec les groupes sociaux» (Goffman, 1972, p. 8). Selon Goffman, «la plupart des groupes, à la différence des rassemblements orientés, continuent d'exister au-delà des occasions où les membres sont physiquement ensemble» (ibid., p. I I). Dès lors, l’action conjointe 
entre individus physiquement co-présents n’est pas d'abord une caractéristique des groupes, mais des rassemblements orientés (ou «systèmes d'activité située») :

Dans la vie de nombreux petits groupes, des occasions surviennent régulièrement où tous les membres et seulement les membres [...] maintiennent conjointement [jointly sustain] un système d'activité située [...]: ils jouent à un jeu [...], ou font une pause-cigarette ensemble. Appeler ces rassemblements des «réunions du groupe» peut facilement induire en erreur et faire penser que l'on étudie le groupe directement [...]. [Mais ces] données initiales concernent les participants à une réunion, pas les membres du groupe (ibid.).

Le problème est donc le suivant : est-il possible, comme le fait Gilbert, d'élaborer une théorie des groupes sociaux à partir d'exemples de rassemblements orientés ? L'exemple paradigmatique de la promenade ensemble n'appartient-il pas d'abord à l'ordre de l'interaction? Cela dit, Goffman soutient que dans certaines conditions, des interactions peuvent créer un groupe (Goffman, 1972, p. I3). Les objections inspirées de Goffman ne sont donc pas forcément fatales à la théorie du sujet pluriel, d'autant plus que l'on peut accepter cette théorie sans pour autant penser qu'elle est la clé menant aux groupes sociaux ${ }^{17}$.

Le philosophe Hans Bernhard Schmid met également en doute le lien entre sujets pluriels et groupes sociaux, en posant la question de savoir si l'identification réflexive est suffisante à la constitution d'un groupe. Selon Gilbert, il suffit que des individus aient conscience d'appartenir à un sujet pluriel, dans les conditions de savoir commun, pour que le groupe existe. Elle rejoint en cela Simmel, pour qui l'unité des entités sociales n'est rien de plus que «la conscience de former une unité avec les autres» (Simmel, cité par Gilbert, I989, p. I4 $\left.6^{18}\right)$. Or, selon Schmid, «la conscience de l'unité ne fait pas encore l'unité » (Schmid, 20ı, p. 205). Imaginons en effet un cercle d’amis qui, au fil des années, se voient de plus en plus rarement en raison des carrières et des vies familiales de chacun. Malgré cette rareté de plus en plus marquée des

17 Ainsi, Conein ou Orléan s'appuient sur Gilbert sans reprendre sa thèse sur les groupes; Bouvier ou Kaufmann s'appuient sur Gilbert tout en insistant sur le fait que les groupes ne sont pas forcément des sujets pluriels, etc.

18 Voir Simmel, 2002 (1908), p. 64. Mais encore une fois, on peut penser que cette lecture de Simmel est partielle. 
retrouvailles, «tous s'identifient très fortement avec ce groupe et tous savent à quel point est significative l'appartenance à ce groupe pour l'image de soi de tous les autres » (ibid., p. 204). Après une longue période,

se tient enfin de nouveau une réunion. Elle est pour tous un événement évidemment assez important, et tous savent qu'il en va de même pour les autres [...]. Enfin vient le jour dit. Mais cette fois rien ne se déroule comme prévu [...]. L’ambiance habituelle ne se fait pas sentir, la conversation ne s'enclenche pas vraiment, malgré l’effort généralisé; tout simplement, cela ne fonctionne plus (ibid.).

Ici, malgré la conscience subjective de former un groupe, malgré la volonté de se «proclamer communauté», il n'y a plus vraiment de groupe au sens fort, en tout cas pas de «communauté réelle» (ibid.). Le co-engagement à dire «nous » n’est donc pas suffisant pour qu'existe ce groupe, qui semble se réduire en fait à une (ultime ?) rencontre, au sens de Goffman ${ }^{19}$.

\subsection{LA QUESTION DU HORS-GROUPE}

Un autre problème, déjà aperçu supra (section I.3.3) est que Gilbert ne prend guère en compte le hors-groupe. Pourtant, un groupe se constitue en référence à ce qui lui est extérieur : souvent, «l'important n'est pas que A veut être ami avec B mais que $\mathrm{A}$, observant $\mathrm{C}$, veut être ami avec B qui est alors un allié potentiel face à une partie tierce» (Conein, 2007, p. 215). Si le phénomène de l'action conjointe est étayé par la psychologie évolutionniste (voir section I.I), l'importance du hors groupe l'est aussi: de nombreux spécialistes s'accordent pour dire que les primates se lient en coalition car c'est leur «principal instrument de survie sociale dans des systèmes sociaux constitués de larges groupes. La clôture du groupe a lieu pour éviter le harcèlement et le surpeuplement» (ibid.). Les capacités cérébrales des primates leur permettent de traiter les informations socialement pertinentes (identification de la hiérarchie, de l'appartenance, des alliances, etc.) pour créer de tels liens. C'est que les contraintes sociales ont été aussi (voire plus) sélectives que les contraintes posées par l'environnement physique. Un indice allant dans ce sens est que «la taille relative du néocortex

19 Schmid (2010, p. 206) développe un argument symétrique, selon lequel l'identification réflexive n'est pas nécessaire à l'existence d'une communauté. 
des primates [est] corrélée à la taille de leur groupe social » (Clément, Kaufmann, 20II, p. 356). Selon cette perspective, les groupes ne sont pas formés par l'activité conjointe, mais par un travail de reconnaissance des régularités sociales elles-mêmes liées à «un système [cognitif] destiné à surveiller (keeping track of) ces régularités» (Conein, 2007, p. 210). La perspective de Gilbert, mais aussi de Simmel ou Tomasello, serait alors trop focalisée sur la coopération au détriment de la coalition - même si les deux perspectives peuvent être réconciliées puisque la coopération permet de maintenir le groupe coalitionnel (ibid., p. 217) ${ }^{20}$.

Enfin, l'incomplétude de la théorie du sujet pluriel sur la question du hors-groupe se ressent bien lorsqu'on la confronte à certaines intuitions de Goffman, qui insiste sur la capacité du hors-groupe à «ancrer» le processus de formation de groupe :

Paradoxalement [...], si un rassemblement orienté est amené à générer par lui-même un groupe [...], alors il peut être pertinent d'inviter un étranger ou deux - et c'est ce qui se fait soigneusement en certaines occasions sociables. Ces personnes ancrent la formation de groupe qui a lieu, en l'empêchant de se calquer à nouveau sur les relations et les groupes qui existaient précédemment parmi les participants (Goffman, 1972, p. I3).

Hélas, Gilbert n'intègre pas du tout ce genre d’idée dans sa conceptualisation : pour elle, la formation de groupe est un processus exclusivement interne.

\subsection{LES GROUPES PEUVENT-ILS VRAIMENT AVOIR DES CROYANCES?}

Une troisième catégorie de critiques questionne le holisme de Gilbert. Pour le philosophe Anthonie Meijers, un groupe ne peut pas avoir de croyances au sens strict. Néanmoins, il ne s’agit pas de remettre en cause la nécessité de dépasser la conception «sommative» des croyances collectives, selon laquelle un groupe croit que p si une majorité de ses membres croit que p. Cette conception n'est en effet pas valable pour des attributions de croyance du type : «le cabinet d'expertise croit que l’alimentation génétiquement modifiée ne présente pas de danger et ne devrait

20 L'importance du hors-groupe est également soulignée par Frédéric Vandenberghe, qui pointe certaines limites de la réflexion gilbertienne. Mais l'argument de l'auteur tourne court. En effet, son analyse de la formation des collectifs est d'une obscurité extrême. Il y aurait une «connexion entre les âmes», des «énergies fusionnelles», une conscience supra-individuelle «s'écoulant à travers» les membres des groupes... (Vandenberghe, 2010, p. 254, 26I). 
pas être interdite» (Meijers, 2002, p. 73). En effet, les membres du cabinet «sont censés ne pas changer leur point de vue sur l'alimentation génétiquement modifiée tant qu’une procédure ne sera pas mise en œuvre par le cabinet» (ibid.). La conception sommative ne rend pas compte de cet élément normatif. La tentative de Gilbert est donc nécessaire. Mais elle tend, selon Meijers, à attribuer trop vite aux groupes des états mentaux qui ne peuvent être portés que par des individus.

En effet, les croyances individuelles peuvent être tout à fait involontaires, tandis que les croyances de groupe ne le sont jamais vraiment: «je ne peux pas décider de croire que je ne mourrai jamais [...] tandis que les croyances collectives [au sens fort] requièrent une sorte d’assentiment, d'accord, ou de décision volontaires par les membres du groupe pour que la croyance devienne collective » (Meijers, 2002, p. 77). Par ailleurs, les croyances de groupe ne sont jamais purement épistémiques (elles ne visent jamais uniquement la vérité), notamment parce qu'elles dépendent d'un certain contexte: une personne peut croire que p est vrai - par exemple, «la terre est plate»-indépendamment du contexte dans lequel elle se trouve, alors qu'un groupe ne peut avoir de croyance qu'en lien avec «une situation, un rôle» (ibid.). Certes, un groupe peut croire épistémiquement que la terre est ronde, mais il s'agit alors forcément d'une croyance sommative («tout le monde dans le groupe croit que la terre est ronde»), et non collective au sens fort («nous, en tant que groupe, croyons que la terre est ronde »). Par conséquent, Meijers propose de dire que si les individus ont des croyances, les groupes ne peuvent avoir que des acceptances, c'est-à-dire des états mentaux I) ne visant pas uniquement la vérité ; 2) dépendants du contexte ${ }^{21}$.

Ces critiques sont constructives : elles ne visent tant pas à réfuter la théorie du sujet pluriel qu’à la complexifier. Cependant, Gilbert demeure réticente à remplacer la notion de croyance par celle d'acceptance: pour elle, les groupes peuvent bel et bien avoir des croyances. Son argument central est que les «rejectionists» (ceux qui pensent que les croyances de groupe ne sont pas vraiment des croyances) considèrent a priori les croyances individuelles comme ayant les propriétés par excellence de la croyance; or, pourquoi le niveau individuel serait-il plus approprié pour rendre compte de ce phénomène? Gilbert propose de faire confiance au langage ordinaire : 
Un critère important pour rendre compte d'un phénomène [...] est le degré de réactivité à l'usage des termes pertinents. Dans le cas présent, les termes centraux sont «croyance» et ses apparentés (croit, croyant, etc.). Rendre compte adéquatement de la croyance suppose alors d'inclure dans l'analyse tous les phénomènes désignés comme étant des cas de «croyance» [...]. Donc, à moins qu'il y ait une bonne raison d'exclure les énoncés renvoyant à des croyances collectives [collective belief statements], il serait mieux d'inclure leurs référents [dans l'analyse] [...], au lieu [...] de considérer seulement les énoncés renvoyant à des croyances individuelles [individual belief statements] et leurs référents (Gilbert, 2002, p. 48).

De ce point de vue, il n'y a pas de raison de dire que les croyances de groupe sont plus contextuelles que les croyances individuelles. Par exemple, si un «comité pour l'amélioration du campus universitaire» croit, à la suite d’un co-engagement de ses membres, qưune brasserie est nécessaire sur le campus, alors la situation dudit comité est similaire à celle d'une personne individuelle qui croit qu'une brasserie est nécessaire sur le campus (ibid., p. 53). Une erreur potentielle serait ici de confondre le contexte dans lequel le co-engagement a eu lieu et le contenu dudit co-engagement: «ce que le comité croit [...] est déterminé par le contenu du co-engagement constitutif. Dans le cas en question, le co-engagement est simplement de croire que $p$ tel un seul corps (as a body). Il n'y a aucun renvoi, explicite ou implicite, au contexte» (ibid.). De même, il n'y a pas de raison de dire que les croyances de groupe sont toujours voulues par le groupe. Certes, elles sont constituées par une volonté collective des membres du groupe. Mais les croyances individuelles sont similaires : si un individu, dans certains cas, ne peut pas vouloir croire (believe at will), il reste que croire nécessite un usage de la volonté, par exemple celle de juger (ibid., p. 60). Une considération «symétrique» des croyances individuelles et collectives requiert donc d’accorder aux groupes les propriétés de la croyance au sens strict.

Une autre critique consiste à montrer que la condition de savoir commun («chacun sait que chacun est co-engagé ») est parfois difficile à réaliser, ce qui jette le doute sur la réalité des sujets pluriels. Dans le cas de la conférence de Bohr à Côme (supra, section I.3.2), le co-engagement est implicite, mais il a lieu à un moment suffisamment saillant pour que les membres de l'école de Copenhague comprennent qu'ils sont désormais co-engagés. Toutefois, dans les sciences, il arrive qu'un co-engagement naisse progressivement du simple fait de «travailler 
ensemble » : c'est ainsi que l'économiste Ludwig von Mises, après avoir travaillé étroitement avec son étudiant Fritz Machlup, se mit à penser qu'un co-engagement avait implicitement émergé entre eux (Bouvier, 2010, p. 193). Lorsque Machlup exprima une opinion différente de celle de Mises à propos du principe de rationalité, ce dernier «exprima une indignation si forte qu’il refusa de parler à Machlup durant plusieurs années » (ibid.). Mais Machlup ne pensait pas qu'il était co-engagé : s'il s'est senti coupable d'avoir contredit Mises, c'est seulement après-coup, une fois qu’il réalisât que Mises pensait que lui, Machlup, était co-engagé à soutenir les mêmes thèses. La condition de savoir commun n'est donc pas réalisée: bien qu'il y ait eu rupture de contrat (du moins subjectivement, puisque Mises est indigné et Machlup se sent coupable), il n’y avait pas de sujet pluriel. Or, il y a des raisons de penser que ce genre de situation - où deux individus ont une interprétation différente de l'engagement qui les lie - est fréquent, du moins dans la vie scientifique. Ce qui peut amener à être «très sceptique à propos de l'existence de groupes dans le sens de Gilbert, c'est à-dire comme "sujets pluriels", du moins dans les sciences » (ibid., p. 195).

\section{CONCLUSION}

Au terme de ce parcours, il apparaît que l'œuvre de Gilbert a un authentique écho dans les sciences sociales, même si chaque discipline prise isolément semble encore assez peu concernée par la théorie du sujet pluriel. Celle-ci semble avoir été remarquée surtout par des social scientists ayant une orientation résolument interdisciplinaire: Bouvier, Conein et Kaufmann pour leur dialogue avec les sciences cognitives et la philosophie, Orléan pour son dialogue avec la sociologie, Tomasello pour son dialogue avec la philosophie sociale, etc. Il est également remarquable que dans le volume «Qu'est-ce qu'un collectif» de Raisons pratiques - revue sociologique particulièrement ouverte aux autres disciplines -, plus de la moitié des contributions (sept sur treize) font référence à Gilbert (Kaufmann et Trom, 20ıo). Incontestablement, certains points de la théorie du sujet pluriel sont fragiles. Mais son idée centrale, une fois aménagée, amendée et complexifiée, semble devenir de plus en plus intéressante pour les sciences sociales. 


\section{BIBLIOGRAPHIE}

BATIFOULIER Ph. (dir.), 200I, Théorie des conventions, Paris, Economica.

BELLER M., 1999, Quantum Dialogue: The Making of a Revolution, Chicago, University of Chicago Press.

BOUDON R., 1995, Le Juste et le Vrai, Paris, Fayard.

BOUVIER A., 2002, «Un modèle polyphonique en épistémologie sociale. Croyances individuelles, pluralité des voix et consensus en matière scientifique», Revue européenne des sciences sociales, 40-3, p. 39-58.

-, 2004, «Individual Beliefs and Collective Beliefs in Sciences and Philosophy. The Plural Subject and the Polyphonic Subject Accounts: Case Studies», Philosophy of the Social Sciences, 34-3, p. 382-407.

-, 2010, «Passive Consensus and Active Commitment in the Sciences», Episteme, 27-3, p. 185-197.

BRATMAN M., 2009, «Modest Sociality and the Distinctiveness of Intention», Philosophical Studies, 144, p. 149-165.

CARASSA A., COLOMBETTI M., 2009, «Joint meaning», Journal of Pragmatics, 4I-9, p. 1837-1854.

-, 2011, «Layers of Joint Commitments in Interpersonal Communication», Proceedings of CogSci 2011 (33 ${ }^{\text {rd }}$ Meeting of the Cognitive Science Society), Boston, p. $1055-1060$.

CHEVALLEY C., 1985, «Complémentarité et langage dans l'interprétation de Copenhague», Revue d'histoire des sciences, 38-3/4, p. 251-292.

CLÉMENT F., KAUFMANN L., 20II, «Ouverture: Vers une sociologie cognitive "intégrative" », in La Sociologie cognitive, F. Clément et L. Kaufmann (dir.), Paris, Orphys/Maison des Sciences de l'Homme, p. 355-368.

CONEIN B., 2005, Les Sens sociaux: trois essais de sociologie cognitive, Paris, Economica.

-, 2007, «Group Patterns, Joint Action, and Social Cognition: The Simmelian Hypothesis», Intellectica, 46-47, p. 207-219.

DESCOMBES V., 2000, «The Philosophy of Collective Representations», History of the Human Sciences, I3-I, p. 37-49. 
DURKHEIM É., 2005 (1894), Les Règles de la méthode sociologique, Paris, PUF.

FAVEREAU O., 200I, «Préface», in Théorie des conventions, Ph. Batifoulier (dir.), Paris, Economica, p. 5-8.

GILBERT M., 1989, On Social Facts, London \& New York, Routledge.

-, 1996, Living Together: Rationality, Sociality, and Obligation, Lanham, Rowman \& Littlefield.

-, 2002, «Belief and Acceptance as Features of Groups», Protosociology, 16, p. 35-69.

-, 2003a (1990), Marcher ensemble: Essais sur les fondements des phénomènes collectifs, Paris, PUF.

-, 2003b (1999), «Reconsidérer la théorie du "contrat réel" de l'obligation politique» in M. Gilbert, Marcher ensemble: Essais sur les fondements des phénomènes collectifs, Paris, PUF.

-, 2006, «Croyances collectives» in Dictionnaire des sciences humaines, S. Mesure et P. Savidan, Paris, PUF, p. 225-229.

-, 2008a, «Social Convention Revisited», Topoi, 27, p.5-16.

-, 2008b (2006), «La responsabilité collective et ses implications», Revue française de science politique, 58-6, p. 899-9/3.

GOFFMAN E., 1972, Encounters, London, Penguin.

GRÄFENHAIN M., BEHNE T., CARPENTER M., TOMASELLO M., 2009, «Young Children's Understanding of Joint Commitments», Developmental Psychology, 45-5, p. $1430-1443$.

KAUFMANN L., 2010, «Faire collectif. De la constitution à la maintenance», in Qu'est-ce qu'un collectif?, L. Kaufmann et D. Trom (dir.), p. 331-372.

-, TROM D. (dir.), 20I0, Qu'est-ce qu'un collectif?, Paris, Éd. de l'EHESS, «Raisons Pratiques».

LEWIS D., 2002, Convention: A Philosophical Study, Oxford, Blackwell.

MEIJERS A., 2002, «Collective Agents and Cognitive Attitudes», Protosociology, 16, p. 70-85.

ORLÉAN A., 2004, «L'économie des conventions: définitions et résultats》, in Analyse économique des conventions, A. Orléan (dir.), Paris, PUF, p. 9-48. 
PACHERIE E., 20II, «Framing Joint Action», Review of Philosophy and Psychology, 2-2, p. $173-192$.

SCHMID H.B., 20I0, «Malentendus collectifs. Réflexions sceptiques sur la théorie-type de l'identité politique», in Qu'est-ce qu'un collectif?, L. Kaufmann et D. Trom (dir.), p. 197-219.

SEARLE J., 1982 (1979), Sens et expression, Paris, Minuit.

SIMMEL G., 2002 (1908), Sociologie. Études sur les formes de la socialisation, Paris, PUF.

STAVO-DEBAUGE J., 2010, «Dé-figurer la communauté? Hantises et impasses de la pensée (politique) de J.-L. Nancy», in Qu'est-ce qu'un collectif?, L. Kaufmann et D. Trom (dir.), p. |37-I7I.

TOMASELLO M., (ed.), 2009, Why we cooperate, Cambridge Mass., MIT Press.

TROM D., 2010, «L'organisation de l'expérience des collectifs politiques modernes», in Qu'est-ce qu'un collectif?, L. Kaufmann et D. Trom (dir.), p.373-403.

TUOMELA R., 1992, «Group Beliefs», Synthese, 91-3, p. 285-318.

URRUTIAGUER D., BATIFOULIER Ph., MERCHIERS J., 200I, «Peut-on se coordonner sur une base arbitraire? Lewis et la rationalité des conventions», in Théorie des conventions, Ph. Batifoulier (dir.), Paris, Economica, p. 63-96.

VANDENBERGHE F., 20I0, «Le collectif en action. Des catégories aux groupes en fusion», in Qu'est-ce qu'un collectif?, L. Kaufmann et D. Trom (dir.), p. 249-270.

WEBER M., 1995 (1921), Économie et Société, Paris, Pocket.

WRAY B., 200I, «Collective Belief and Acceptance», Synthese, 129-3, p. 319-333. 